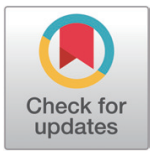

Received: Oct 16, 2021

Revised: Nov 27, 2021

Accepted: Nov 30, 2021

\#These authors contributed equally to this work.

${ }^{*}$ Corresponding author

Sung-Jo Kim

Division of Cosmetics and

Biotechnology, College of Life and

Health Sciences, Hoseo University,

Asan 31499, Korea.

Tel: +82-41-540-5571

E-mail: sungjo@hoseo.edu

Ki-Duk Song

Department of Agricultural

Convergence Technology, Jeonbuk

National University, Jeonju 54896,

Korea.

Tel: +82-63-219-5523

E-mail: kiduk.song@jbnu.ac.kr

Copyright $\odot 2022$ Korean Society of Animal Sciences and Technology.

This is an Open Access article distributed under the terms of the

Creative Commons Attribution

Non-Commercial License (http://

creativecommons.org/licenses/by-

nc/4.0/) which permits unrestricted

non-commercial use, distribution, and

reproduction in any medium, provided

the original work is properly cited.

\section{Suppression of the Toll-like receptors 3 mediated pro- inflammatory gene expressions by progenitor cell differentiation and proliferation factor in chicken DF-1 cells}

\author{
Eunmi Hwang ${ }^{1 \#}$, Hyungkuen Kim ${ }^{1 \#}$, Anh Duc Truong ${ }^{2}$, Sung-Jo Kim ${ }^{1 *}$ and \\ Ki-Duk Song ${ }^{2 *}$ \\ ${ }^{1}$ Division of Cosmetics and Biotechnology, College of Life and Health Sciences, Hoseo University, Asan \\ 31499, Korea \\ ${ }^{2}$ Department of Agricultural Convergence Technology, Jeonbuk National University, Jeonju 54896, \\ Korea
}

\begin{abstract}
Toll-like receptors (TLRs), as a part of innate immunity, plays an important role in detecting pathogenic molecular patterns (PAMPs) which are structural components or product of pathogens and initiate host defense systems or innate immunity. Precise negative feedback regulations of TLR signaling are important in maintaining homeostasis to prevent tissue damage by uncontrolled inflammation during innate immune responses. In this study, we identified and characterized the function of the pancreatic progenitor cell differentiation and proliferation factor (PPDPF) as a negative regulator for TLR signal-mediated inflammation in chicken. Bioinformatics analysis showed that the structure of chicken PPDPF evolutionarily conserved amino acid sequences with domains, i.e., SH3 binding sites and CDC-like kinase 2 (CLK2) binding sites, suggesting that relevant signaling pathways might contribute to suppression of inflammation. Our results showed that stimulation with polyinosinic:polycytidylic acids (Poly $[l: C]$ ), a synthetic agonist for TLR3 signaling, increased the mRNA expression of PPDPF in chicken fibroblasts DF-1 but not in chicken macrophage-like cells HD11. In addition, the expression of pro-inflammatory genes stimulated by Poly(l:C) were reduced in DF-1 cells which overexpress PPDPF. Future studies warrant to reveal the molecular mechanisms responsible for the anti-inflammatory capacity of PPDPF in chicken as well as a potential target for controlling viral resistance.
\end{abstract}

Keywords: Chicken, DF-1, Innate immunity, Inflammation, Toll-like receptor 3, Pancreatic progenitor cell differentiation and proliferation factor 
ORCID

Eunmi Hwang

https://orcid.org/0000-0001-8166-384X

Hyungkuen Kim

https://orcid.org/0000-0001-7508-9933

Anh Duc Truong

https://orcid.org/0000-0002-2472-8165

Sung-Jo Kim

https://orcid.org/0000-0003-4590-3644

Ki-Duk Song

https://orcid.org/0000-0003-2827-0873

\section{Competing interests}

No potential conflict of interest relevant to

this article was reported.

Funding sources

This work was carried out with the support of the Cooperative Research Program for Agriculture Science and Technology Development (Project No. PJ015612), Rural Development Administration, Republic of Korea, and the Basic Science Research Program" through the National Research Foundation of Korea (NRF) funded by the Ministry of Education (2021R111A3057071).

Acknowledgements

Not applicable.

Availability of data and material Upon reasonable request, the datasets of this study can be available from the corresponding author.

\section{Authors' contributions}

Conceptualization: Kim SJ, Song KD.

Methodology: Hwang E, Kim H.

Investigation: Hwang $\mathrm{E}, \mathrm{Kim} \mathrm{H}$.

Writing - original draft: Hwang E, Kim H Writing - review \& editing: Hwang E, Kim H, Truong AD, Kim SJ, Song KD.

Ethics approval and consent to participate The study followed institutional rules for the care and use of laboratory animals and a protocol approved by the Ministry of Agriculture and Rural Development of Vietnam (TCVN 8402:2010 and TCVN 840026:2014).

\section{INTRODUCTION}

Innate immunity, known as a host defense system, recognizes and eliminates pathogens. Toll-like receptors (TLRs) play a key role in detecting pathogens, such as bacteria, viruses, and chemicals as well as activating innate immunity [1,2]. TLR3 activates the inflammatory pathway by recognizing double-stranded RNA produced by viral infection. [3,4]. Inflammation maintains homeostasis and prevents further infection, but excessive inflammation also damages normal cells [5]. TLR3 activation by highly pathogenic avian influenza A H5N1 virus, pandemic H1N1 virus, and dsRNA analog polyinosinic:polycytidylic acids (Poly[I:C]) induces inflammation and impairment of lung function, and knockout of TLR3 was positive for improvement of lung innate immunity and survival rate $[6,7]$. Therefore, TLR3 inhibition is a good target for improving the survival rate of chickens against respiratory infectious dsRNA virus disease. Functional feed additives such as alpha-lipoic acid (ALA) inhibit TLR3 activity and improve chicken meat quality and productivity, but are not practical due to their high cost [8,9]. In chickens, it is an attractive goal to genetically understand the mechanisms of TLR3 regulation and increase its regulatory efficiency. TLR regulatory mechanisms are well established in humans, but do not apply equally in chickens. In the case of human TLR4, MD-2 protein acts as an activity inhibitor of TLR4, whereas chicken TLR4 requires complex formation with MD-2 protein for pathogen detection [10]. In the case of TLR3, the amino acid sequence of the intracellular region, Toll/interleukin-1 receptor (TIR), is markedly different in birds and primates (Fig. 1). The regulatory mechanisms of chicken TLR3 signaling remain to be studied.

In this study, we identified the pancreatic progenitor cell differentiation and proliferation factor (PPDPF) as a potential inflammatory regulator in the chicken. PPPDF is a key modulator of exocrine pancreatic development and transcriptional target of retinoic acid (RA) and pancreas transcription factor 1a (PTF1A) [11,12]. In zebrafish, PPDPF has been shown to promote pancreatic exocrine gland growth and differentiation while inhibiting pancreatic endocrine gland growth and secretion. PPDPF gene is highly overexpressed in ovarian cancers and upregulated DNA replication pathway [13]. Furthermore, the PPDPF gene was discovered in prostate tumors as a genomic marker that can be utilized to predict biochemical recurrence [14]. Liver-specific PPDPF overexpression effectively inhibits high-fat diet (HFD)-induced mechanistic target of rapamycin (mTOR) signaling activation and hepatic steatosis in mice [15]. We investigated the levels of PPDPF expression in chicken organs and TLR3-activated chicken cells, as well as the role of PPDPF in TLR3 expression and activation in chicken cells. We also explain the diversity of PPDPF across the species and suggest chicken PPDPF as a factor for TLR3 activation and inflammation.

\section{MATERIALS AND METHODS}

\section{Bioinformatics of progenitor cell differentiation and proliferation factor}

PPDPF protein amino acids sequences, i.e., chicken (Gallus gallus; XP_027329124.1), duck (Anas platyrhynchos; XP_027329124.1), human (Homo sapiens; Q9H3Y8.1), porcine (Sus scrofa; XP_020933363.1), bovine (Bos taurus; NP_001068762.1), equine (Equus caballus; XP_023482697.1), murine (Mus musculus; Q9CR37), and feline (Felis catus; XP_003983360) were retrieved from National Center for Biotechnology Informaion, U.S. National Library of Medicine, USA. Predicted phosphorylation site was evaluated using NetPhos-3.1 service (DTU Health Tech, Lyngby, Denmark) [16]. Predicted ubiquitination site was evaluated using RUBI Version 1.0 (BioComputing UP, Padua, Italy) [17] or obtained from neXtProt database (Swiss Institute 
Species (accession no.)

Gallus gallus (ADZ48550.1)

Anas platyrhynchos (AlW60885.1)

Homo sapiens (AAH96335.1)

Pan troglodytes (BAG55029.1)

Gallus gallus (ADZ48550.1)

Anas platyrhynchos (AIW60885.1)

Homo sapiens (AAH96335.1)

Pan troglodytes (BAG55029.1)

\section{Amino acid sequences of TIR domain}

ydayvihark dtnwvltnft tleeneqfqv kfcleerdfe agisefeaii ncirrsrkii 60

ydayilharh drnwvlknfi sleknkqfev rfcleerdfe agvsefeaii nsirrsrkii $\quad 60$

yihaykdkd wVwehfssme kedqslkfcl eerdfeagvf eleaivnsik rsrkiifvilt 60

Yiihaykdkd wVwehfssme kedqslkfcl eerdfeagvf eleaivnsik rsrkiifvi t $\quad 60$

fivtehllqd pwcrkfkvhh alqqaleqs dsiiliflhn iqdyk 105

fvvtehllk pwckkfkvyh avqqaieqsr dsiilvflhd iqdyklnhal clrrgmfrsr cilkwpaq 128

hhllkdplck rfkvhhavqq aieqnldsii lvfleeipdy k 101

hhllkdpvck rfkvhhavqq aieqnldsii lvfleeipdy k 101

\section{$100 \% \quad \leq 50 \%$}

Fig. 1. TIR amino acid sequences. Amino acid sequences of intracellular domain of TLR3 of chicken (Gallus gallus, ADZ48550.1), duck (Anas platyrhynchos, AIW60885.1), human (Homo sapiens, AAH96335.1), and chimpanzee (Pan troglodytes, BAG55029.1). Homology of amino acid sequences are highlighted as black (100\%) or gray ( $\leq 50 \%)$. TIR, toll/interleukin-1 receptor.

of Bioinformatics, Lausanne, Switzerland). The structure of chicken PPDPF was predicted using IntFOLD server [18,19]. 3-dimensional (3D) structure was constructed by using PyMol software (Schrödinger, New York, NY, USA).

\section{Cell culture condition}

DF-1 chicken embryonic fibroblast cell line (Cat\# CRL-12203, ATCC, Manassas, VA, USA) and the HD-11 chicken macrophage-like cell line [20] were cultured with Dulbecco's modified Eagle's medium (DMEM; Cat\# 10-013-CVR, Corning, Corning, NY, USA) supplemented with $1 \%$ penicillin-streptomycin and $10 \%$ fetal bovine serum (FBS; Invitrogen, Carlsbad, CA, USA) in a $5 \% \mathrm{CO}_{2}$ atmosphere at $37^{\circ} \mathrm{C}$. The HD-11 cell line was provided by Dr. Hyun S. Lillehoj at the Agricultural Research Services (ARS) at the United States Department of Agriculture (USDA), Beltsville, Maryland, USA.

\section{Chicken tissue collection}

The specific-pathogen-free White Leghorn chickens (4-weeks old) were purchased from the Poultry Research Centre of the National Institute of Animal Science (NIAS; Hanoi, Vietnam). The chickens were given unlimited access to antibiotic-free feed and water. A total of 5 tissue samples were collected from the chickens, and placed in liquid nitrogen for total RNA extraction. All the experiments were conducted in compliance with the institutional rules for the care and use of laboratory animals, as well as implementing the protocol approved by the Ministry of Agriculture and Rural Development of Vietnam (TCVN 8402:2010 and TCVN 8400-26:2014).

\section{RNA isolation and cDNA synthesis}

Total RNA was isolated from the DF-1 cells, HD-11 cells, and lung tissue of White Leghorn chickens using a TRIzol reagent (Cat\# 15596018, Invitrogen) according to the manufacturer's instructions. cDNA was synthesized using WizScript cDNA Synthesis Kit (Cat\# W2202, Wizbiosolutions, Seongnam, Korea).

\section{Real-time polymerase chain reaction}

Real-time PCR was performed using StepOnePlus Real-Time PCR System (Applied Biosystems, Foster City, CA, USA) with 10 ng cDNA, SYBR Green qPCR Master Mix (Cat\# DQ385-40h, Biofact, Daejeon, Korea), and $1 \mathrm{pM}$ primer (Table 1). Levels of TLR3, tumor necrosis factor (TNF) 
Table 1. Primer sequences for DF-1 chicken embryonic fibroblasts

\begin{tabular}{lll}
\hline \multicolumn{1}{c}{ Gene } & \multicolumn{1}{c}{ Sequences (5' to 3') } & \multicolumn{1}{c}{ Accession No. } \\
\hline TLR3 & F: AAAGTGGGGCATTTTCCTCCT & NM_001011691.3 \\
RF-KB1 & F: TTGAGACCTTCGATTCATGACC & \\
& R: TGTGTTGTCCTCCTGGTATATCT & NM_205134.1 \\
TNFa & F: GGTTCGAGTCGCTGTATCAGG & MF000729.1 \\
& R: ACTCCCACCACCCCAAAATA & MF801626.1 \\
COX2 & F: TAAAGCTTACGGCCATGGGG & NM_001167719.1 \\
& R: AGTGTCCTTCACTGTTGGTGG & NM_001167718.1 \\
TRAF3 & F: TCAAGACCAGCTCTCAGCAG & XM_004936343.3 \\
& R: GAACTTTGCTAGTCTGCCCG & (Transcript variant X1 X8, X10 X12) \\
TICAM1 & F: CACATCTGCTCAGGTGGGTC & NM_001081506.1 \\
& R: GGATGATGATGGAACGGGCA & \\
& F: TGCTACGTCGCACTGGATTT & NM_205518.1 \\
\hline
\end{tabular}

TLR3, Toll-like receptors 3; NF-KB1, nuclear factor-kB1; TNFa, tumor necrosis factor $\alpha$; COX2, cyclooxygenase2; TRAF3, TNF receptor-associated factor 3; TICAM1, Toll-like receptor adaptor molecule 1; F, forward; R, reverse.

receptor-associated factor 3 (TRAF3), Toll-like receptor adaptor molecule 1 (TICAM1), nuclear factor$\kappa B 1(N F-\kappa B 1), T N F \alpha$, and cyclooxygenase2 (COX2) mRNA were measured [21]. mRNA foldchange was normalized to $\beta$-actin mRNA using $2^{-\Delta \Delta C t}$ method [22].

\section{Cloning of chicken PPDPF}

cDNA from lung tissue of White Leghorn chickens was used for PPDPF cloning. PCR was performed for isolation and cloning of $P P D P F$ using $P f u$ DNA polymerase with specific primers (Table 2) under the following conditions: $95^{\circ} \mathrm{C}$ for $5 \mathrm{~min}, 30$ cycles of $95^{\circ} \mathrm{C} 30 \mathrm{sec}, 56^{\circ} \mathrm{C} 45 \mathrm{sec}$, $72^{\circ} \mathrm{C} 50 \mathrm{sec}$, and the final extension for $10 \mathrm{~min}$ at $72^{\circ} \mathrm{C}$. Newly synthesized DNA fragments were purified using Gel Purification kit (Cat\# K-303501, Bioneer, Daejeon, Korea). DNA fragments and pEGFP-N3 vectors were digested using HindIII and BamHI restriction enzymes. DNA fragment was inserted into the vector using the TOPcloner kit (Cat\# EZ002S, Enzynomics, Daejeon, Korea) and transformed into E. coli DH5 $\alpha$. The plasmid was purified using the Plasmid Mini Extraction Kit (Cat\# K-3030, Bioneer) and clones containing PPDPF coding sequences (CDS) were selected through sequencing (Bioneer). All processes were carried out according to the manufacturer's instructions.

\section{Transfection of PPDPF}

Chicken DF-1 cells were transfected with pEGFP-N3 vectors concentration at $1 \mu \mathrm{g} / 1.0 \times 10^{6}$

Table 2. Primer sequences for chicken PPDPF cloning

\begin{tabular}{llc}
\hline \multicolumn{1}{c}{ Purpose } & \multicolumn{1}{c}{ Sequences (5' to 3') } & Accession No. \\
\hline Isolation & F: CCAGGTTTTCCATCAGCACAA & NM_001197037.1 \\
& R: AAGGGAAGGGCCATTGCAG & \\
Cloning & F: AAGCTTATGGCCTCCATCCCATCGAGC & NM_001197037.1 \\
& R: GGATCCGGATGAGTGCCCAATGCCTGG & \\
\hline
\end{tabular}

Primers for isolation of PPDPF were in outside of CDS.

Primers for cloning containing HindIII (AAGCTT) or BamHI (GGATCC) sequences.

PPDPF, progenitor cell differentiation and proliferation factor; CDS, coding sequences; $F$, forward; $R$, reverse. 
cells using Lipofectamine 3000 transfection reagent (Cat\# L3000008, Invitrogen) according to the manufacturer's instructions.

\section{Fluorescence microscopy}

Cells were washed with phosphate buffered saline (PBS; pH 7.4), fixed in 3.8\% formaldehyde for $15 \mathrm{~min}$, and stained with 4',6-diamidino-2-phenylindole (DAPI, $1 \mu \mathrm{g} / \mathrm{mL}$ ). Fluorescence was visualized using a DMi8 fluorescence microscope (Leica, Deerfield, IL, USA) and LAS X program (Leica). Lightness and contrast of images were processed using Photoshop CC program (Adobe Systems, San Jose, CA, USA).

\section{Cell viability assay}

Cells were cultured in 96-well plates with 10\% (v/v) WST-1 reagent (Cat\# EZ-3000, DoGenBio, Seoul, Korea) for $2 \mathrm{~h}$ and measured the absorbance at $450 \mathrm{~nm}$ using a microplate reader (Sunrise, Tecan, Männedorf, Switzerland).

\section{Statistical analysis}

Results were obtained from three separate experiments $(n=3)$ and analyzed using GraphPad PRISM 8 software (GraphPad Software, San Diego, CA, USA) and Microsoft Excel software (Microsoft, Redmond, WA, USA). The data are expressed as mean \pm SD. The $p$-value was calculated using an analysis method suitable for each experiment and specified in figure legends. Results were considered statistically significant when the $p$-value was $<0.05$.

\section{RESULTS}

The intracellular domain TIR regulates TLR3 activity through adapter protein binding and signaling when TLR3 is activated by ligand [23]. Therefore, we identified the possibility at the amino acid sequence level for differences in the regulation of TLR3 signaling in primates and avian. Amino acid sequences of the TIR domain between two avian species, chickens (Gallus gallus, ADZ48550.1) and ducks (Anas platyrhynchos, AIW60885.1) were very similar. TIR domain sequence homology was also observed between two primates, i.e., human (Homo sapiens, AAH96335.1) and chimpanzees (Pan troglodytes, BAG55029.1). Comparison of the TIR domain sequences between avian and primates revealed very low homology (Fig. 1), suggesting the possibility that TLR3 signaling of avian species may be distinct from that of primates.

\section{Bioinformatics analysis of chicken PPDPF protein}

We performed an amino acid sequence-based analysis to analyze the interspecies diversity of PPDPF proteins and predict their functions. The PPDPF protein sequences were highly conserved up to the 76th amino acid (AA). The predicted ubiquitination site (99Lys) was identified only in human, bovine, equine, murine, and feline sequences (Fig. 2A). The homology of the PPDPF protein sequence to chicken PPDPF averaged only $50 \%$ in the species we investigated (Figs. 2A and B). Amino acid sequence diversity dropped sharply at 77th AA across all species investigated in this study (Figs. 2A and C). To discover the function of chicken PPDPF, the 3D structure of the chicken PPDPF protein (NP_001183966.1) was predicted using the IntFOLD server. In our protein model, chicken PPDPF was identified as a structure with one alpha-helix (Fig. 2D). In the conserved region of PPDPF (1-76AA), phosphorylation sites are located. And the CLK 2 kinase sites, a SH3 domain binding site, and adenosine triphosphate (ATP) / guanosine triphosphate (GTP) binding sites reported in PPDPF were located [11]. In the variable region (77-113AA), 
A

Species (accession no.)

Amino acid sequences

Gallus gallus (NP_001183966.1) Anas platyrhynchos (XP_027329124.1) Homo sapiens (NP_077275.1) Sus scrofa (XP_020933363.1) Bos taurus (NP_001068762.1) Equus caballus (XP_023482697.1) Mus musculus (NP_079874.1) Felis catus (XP_003983360.1) Unmatched sequences

Gallus gallus (NP_001183966.1) Anas platyrhynchos (XP_027329124.1) Homo sapiens (NP_077275.1) Sus scrofa (XP_020933363.1) Bos taurus (NP_001068762.1) Equus caballus (XP_023482697.1) Mus musculus (NP 079874.1) Felis catus(XP_003983360.1) Unmatched sequences

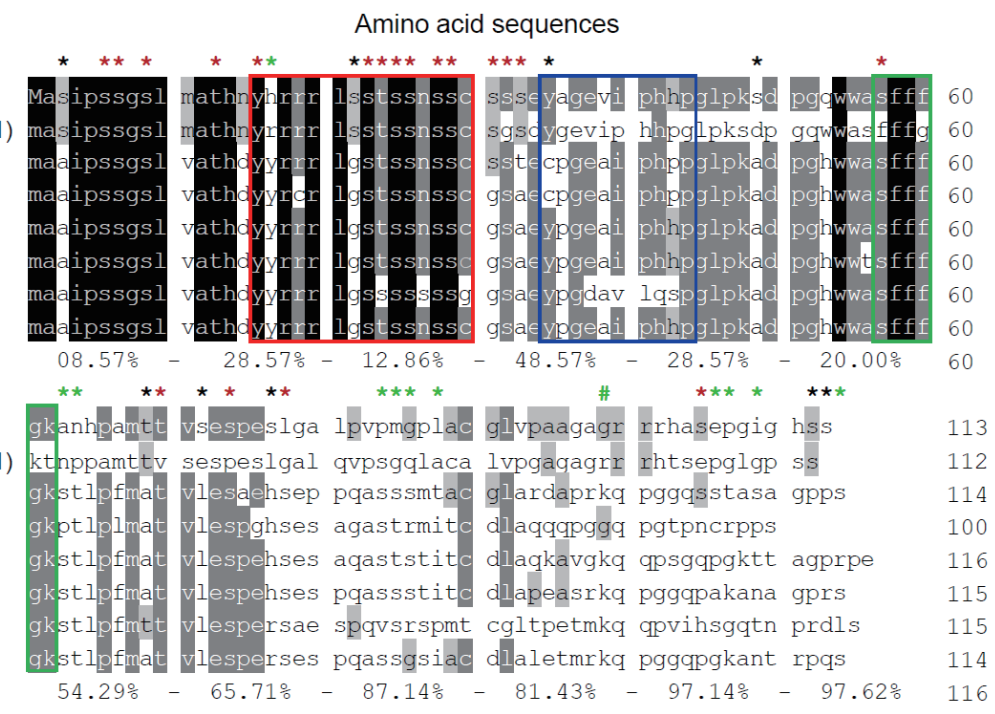

$100 \% \quad<10 \% \quad *$ Predicted phosphorylation sites (Gallus gallus, Homo sapiens, both)

$<70 \% \quad$ \# Predicted ubiquitination site (Gallus gallus, Homo sapiens, both)

$\square$ CLK2 kinase binding site $\square$ sH3 domain binding site $\square$ ATP/GTP binding site

B

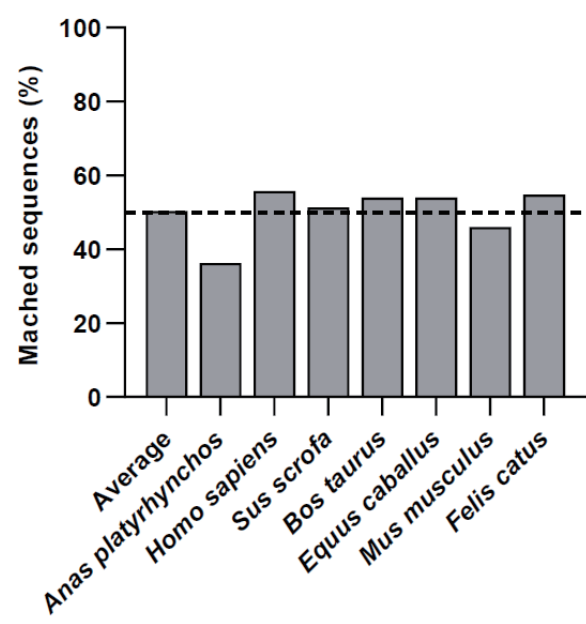

C

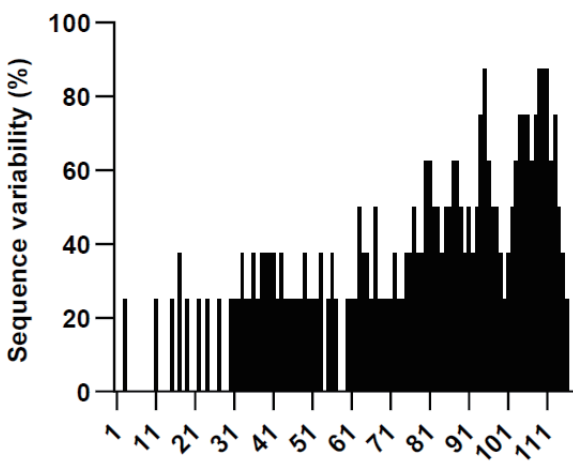

Amino acid position

D

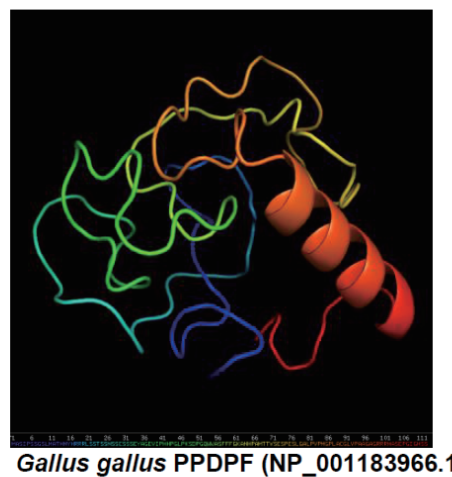

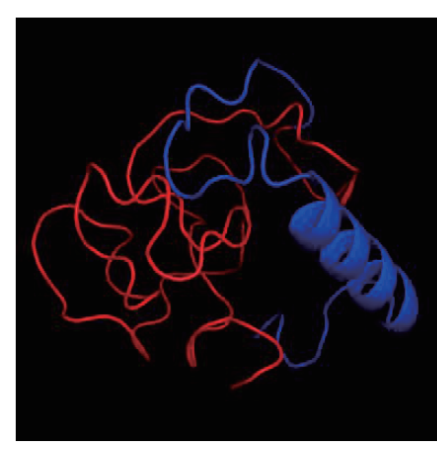

Conversed region (1-76AA) Variable region (77-113AA)

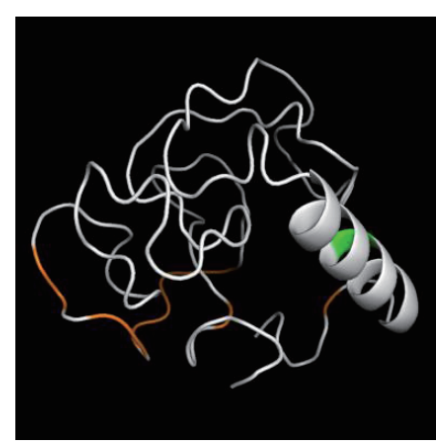

Phosphorylation site (expected) Ubiquitination site (expected)

Fig. 2. Bioinformatics analysis of chicken PPDPF proteins. (A) Amino acid sequences and motif of PPDPF from chicken (Gallus gallus), duck (Anas platyrhynchos), human (Homo sapiens), porcine (Sus scrofa), bovine (Bos taurus), equine (Equus caballus), murine (Mus musculus), and feline (Felis catus). (B) PPDPF amino acid sequence homology to chicken PPDPF protein. (C) Variability of amino acid sequences of PPDPF across the species. (D) Predicted protein structure of chicken (Gallus gallus) PPDPF. CLK2, CDC-like kinase 2; ATP, adenosine triphosphate; GTP, guanosine triphosphate; PPDPF, progenitor cell differentiation and proliferation factor. 
the alpha helix structure and the predicted ubiquitination site were located (Fig. 2D). These results suggest the possibility that PPDPF may play a role that differs from species to species.

\section{PPDPF mRNA expression induced by polyinosinic:polycytidylic acids treatment in} chicken cells

To confirm whether the expression of $P P D P F$ in chickens is affected by dsRNA, PPDPF mRNA fold-change was measured by RT-PCR in Poly(I:C)-treated chicken cell lines. In DF-1 cells, a chicken embryonic fibroblast cell line, $P P D P F$ mRNA expression was increased 25 -fold by treatment with $10 \mu \mathrm{g} / \mathrm{mL}$ Poly(I:C) (Fig. 3A). And, as expected, in chicken macrophage-like cell line HD-11 cells lacking exogenous dsRNA detection ability [24], Poly(I:C) treatment did not affect the expression of PPDPF (Fig. 3B). To evaluate the PPDPF expression levels for each tissue, RT-PCR was performed with cDNA from chicken organs. The expression levels of PPDF were the highest in the lungs and the lowest in the heart among the organs which we investigated (Fig. 3C). Through these results, we confirmed the possibility that $P P D P F$ expression was induced by Poly(I:C), a TLR3 activator, in chickens and is related to the immune response in the lungs.

\section{Effects of PPDPF on the inflammation in DF-1 cells}

To determine the effect of PPDPF expression on TLR3 inflammatory response in chicken cells, we constructed a chicken PPDPF expression vector based on the CDS of PPDPF mRNA (accession no. NM 001197037.1) (Fig. 4A). PCR was used to isolate CDS from chicken PPDPF, which was then introduced into the pEGFP-N3 vector (Figs. 4B and C). Transfection of PPDPF inserted pEGFP-N3 vector into DF-1 cells elevated PPDPF mRNA expression, as validated by RT-PCR (Fig. 4D). DF-1 cells were cultured with Poly(I:C) for 24 hours to examine if PPDPF expression alters the expression of pro-inflammatory genes produced by Poly(I:C), and fluorescence microscopy and RT-PCR were performed (Fig. 5A). The PPDPF protein was uniformly located in the cell including the nucleus. TLR3 signaling genes (TLR3, TRAF3, and TICAM1), as well as pro-inflammatory genes ( $N F-B 1$ and $T N F-\alpha)$, were increased by Poly(I:C) treatment, however PPDPF overexpression suppressed TLR3, TRAF3, TICAM1, and TNF- $\alpha$ expression (Fig. 5C). It is of note that PPDF transfection did not induce toxicity in DF-1 cells (Fig. 5D). These results suggest that overexpression of PPDPF in chicken fibroblasts is a potential TLR3 inhibitor to
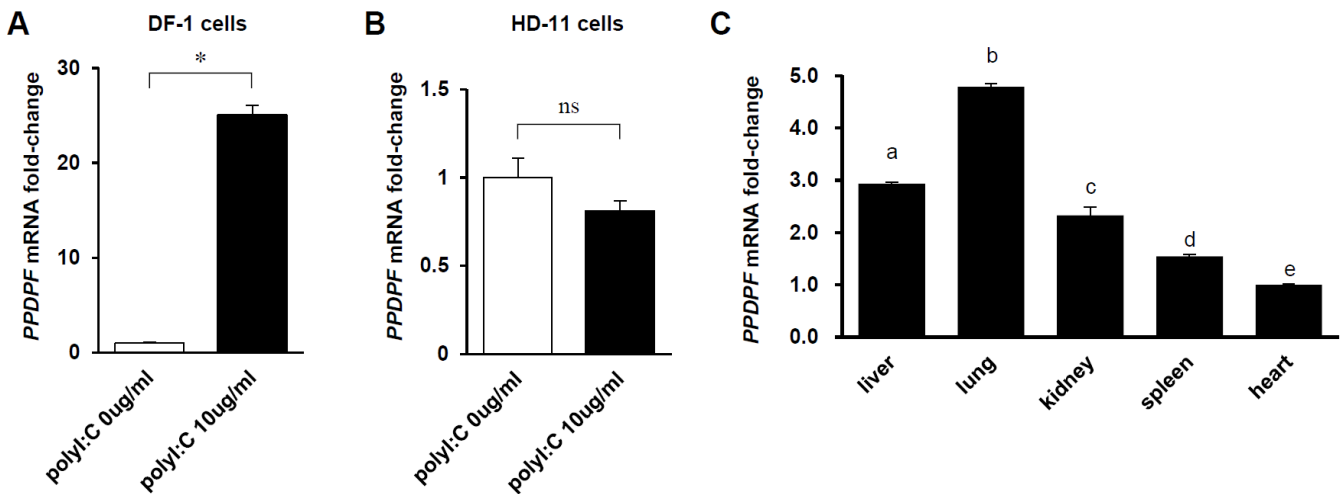

Fig. 3. PPDPF mRNA expression induced by Poly(l:C) treatment in chicken cells. (A, B) PPDPF mRNA expression levels in chicken DF-1 fibroblasts (A) and HD-11 macrophage-like cells (B). mRNA fold-change was normalized to $\beta$-actin mRNA. Data are expressed as mean \pm SD $(n=3)$. ${ }^{*} p<0.01$ and ${ }^{n s} p>0.05$ by unpaired two-tailed Student's $t$-test. (C) mRNA expression level of PPDPF in liver, lung, kidney, spleen, and heart tissue of chicken. mRNA expression levels were measured by real-time PCR. mRNA fold-change was normalized to $\beta$-actin mRNA. Data are expressed as mean $\pm S D(n=3)$. Statistical significance was measured using one-way ANOVA. PPDPF, progenitor cell differentiation and proliferation factor; Poly (I:C), polyinosinic:polycytidylic acids; PCR, polymerase chain reaction. 
A

Gallus gallus PPDPF mRNA sequences (CDS)

1 gcgccgcgge tcaggccgtg cggagcggac gcgggtgctg cgctccetcg ggcggggget

61 tcgagcgcce cgccgggecc ctttaccetc ccetttctet tecggaccec actgggecct

181 atcgagcggc tcgcttatgg ccacgcacaa ctatcaccga aggcgtctga gctccacatc

241 cagcaacagc tcctgcagta gctctgagta tgctggggag gtcatccccc accacceggg

301 tctgcccaaa tctgaccccg gecagtggtg ggccagcttc ttctttggga aggccaatca

361 ccctgccatg acaactgtgt cagagtccce agagagcttg ggagegctgc cagtacccat

421 ggggecectg gegtgtggge tggtgecage agcaggagca ggacgeagge ggcatgecag

481 cgagccaggc attgggeact catcctgagc tgcaatggec cttccettcc ctgcagagct

541 gcttgcagat gtatgtgctg ctttggggta agacaaggca gcagcccccc cagcaattcc

601 tacaccagcc tcttactatt tctcaatgca accgtgccat ttctgtgcag attccgtaac

661 acgtttattg actttgtact totacaatgo ctactaataa actcgagcag ccttttagca $721 \mathrm{C}$
121 agtgccggcc aggttttcca tcagcacaaa cccaggaccc ggagccatgg cetccatccc

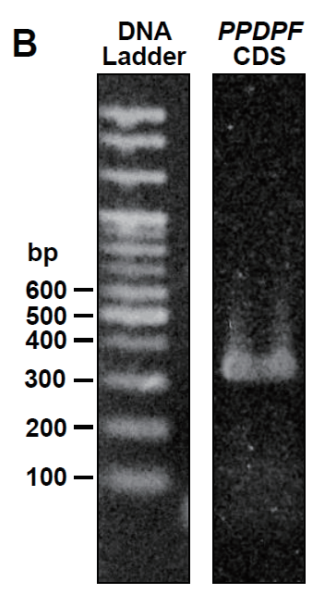

C

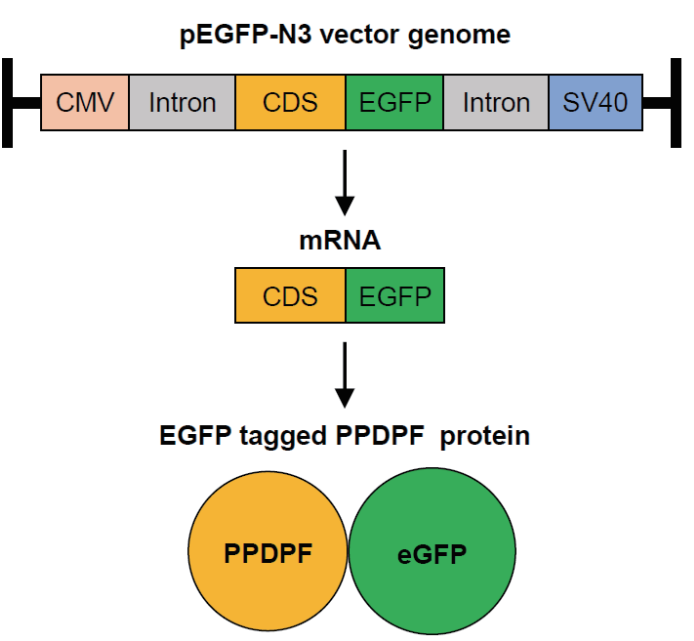

D

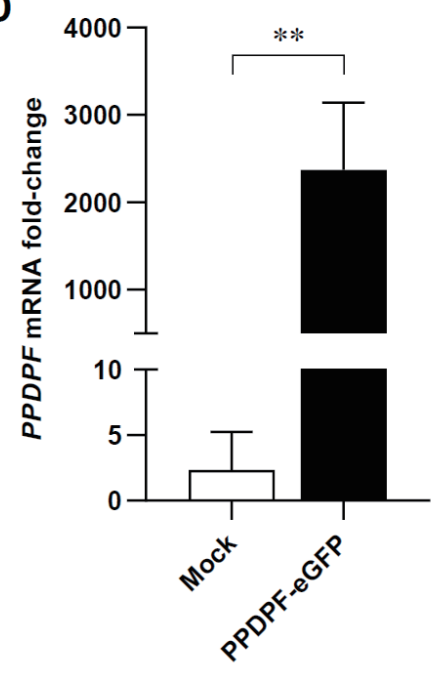

Fig. 4. Construction of PPDPF-eGFP expression vector. (A) Reference sequences of G. gallus PPDPF mRNA (accession no. NM_001197037.1). (B) Fulllength coding sequence (CDS) of chicken PPDPF was amplified using PCR and separated using agarose gel electrophoresis. (C) Design of pEGFP-N3 vector containing CDS of chicken PPDPF mRNA. (D) PPDPF mRNA expression levels in DF-1 cells were measured using RT-PCR after transfected for $48 \mathrm{~h}$. mRNA fold-change was normalized to $\beta$-actin mRNA. Data are expressed as mean $\pm \operatorname{SD}(n=3)$. ${ }^{*} p<0.01$ by unpaired two-tailed Student's $t$-test. PPDPF, progenitor cell differentiation and proliferation factor; PCR, polymerase chain reaction.

downregulate the mRNA expression of TLR3-related genes induced by Poly(I:C). Through the above results, we confirmed that PPDPF inhibits Poly(I:C)-induced TLR3 mRNA expression in DF-1 cells and might act as a negative regulator for dsRNA-induced inflammation.

\section{DISCUSSION}

In chickens, TLR3 is important for immunity against major infectious diseases that threaten the avian industry, such as avian influenza virus (AIV) and Newcastle disease virus (NDV) $[25,26]$. But excessive inflammation might be responsible for reduced chicken productivity $[27,28]$. TLRmediated inflammation against invading pathogens should be dampened to maintain homeostasis to prevent the potential damage resulted from uncontrolled responses. Numerous negative regulatory molecules have been identified and characterized at multiple levels [29]. However, no negative regulators for TLR have been reported in livestock, including poultry. In this study, we discovered PPDPF as a protein capable of suppressing TLR3-mediated inflammatory responses in 
A

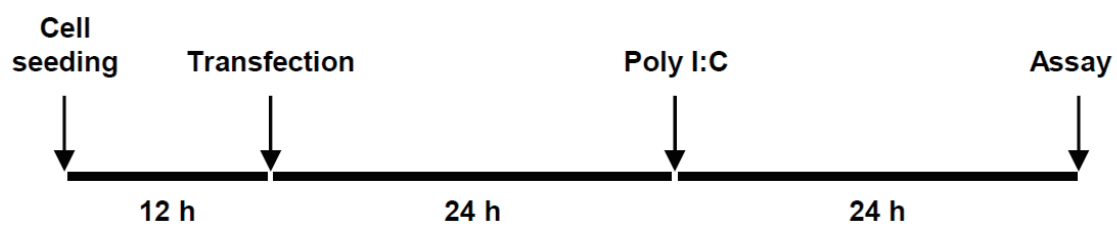

B

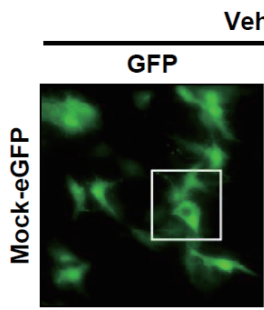

Vehicle
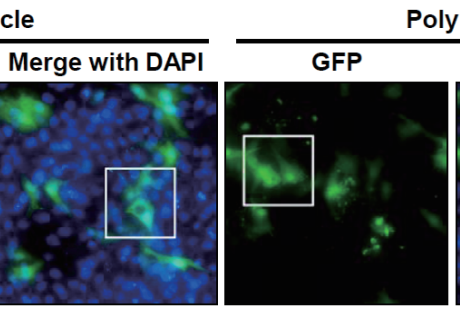

Poly I:C
Merge with DAPI
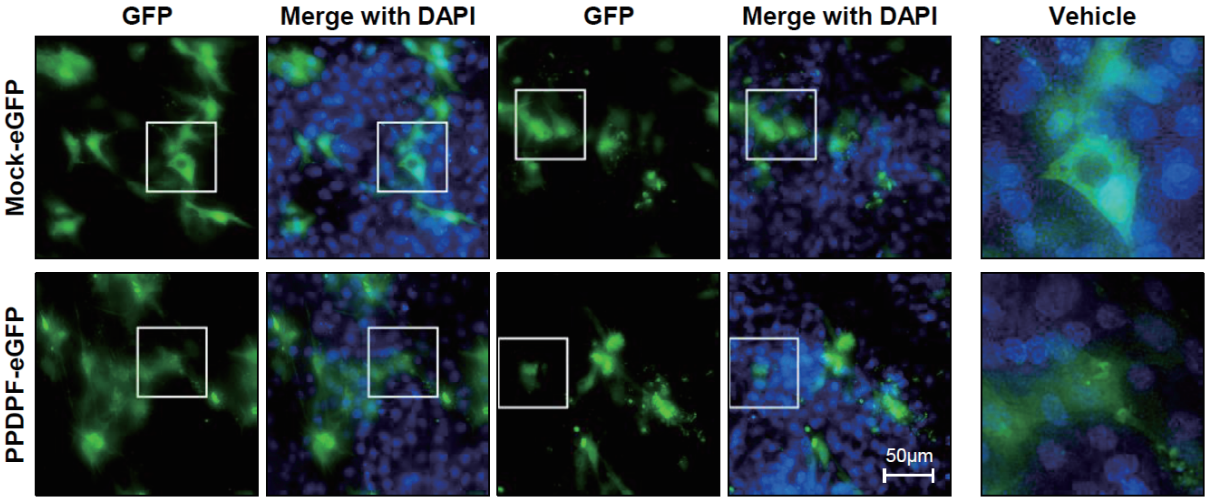

Poly I:C

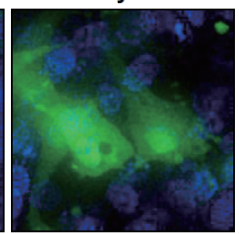

C

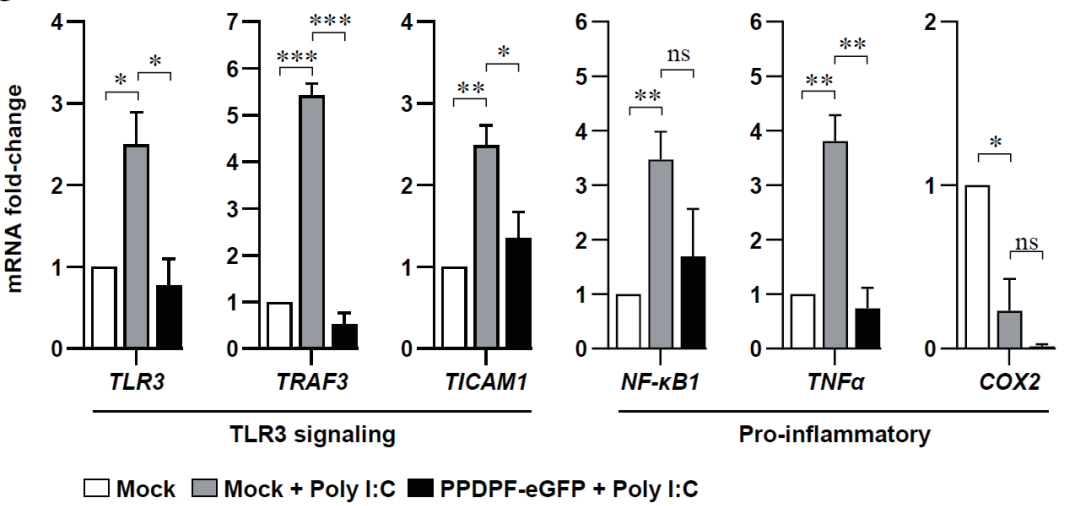

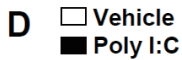

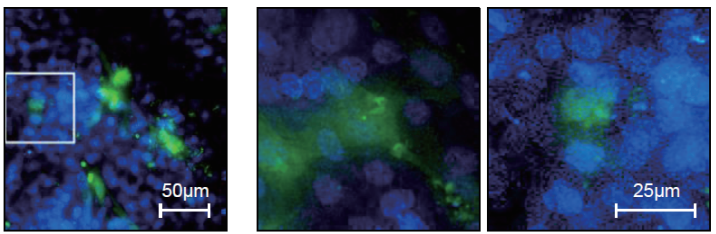

. 5. PPDPF suppresses pro-inflammatory gene expression in poly(l:C) treated DF-1 cells. (A) Experimental design. Poly(l:C) $(10 \mu \mathrm{g} / \mathrm{mL})$ was treated $24 \mathrm{~h}$ after transfection. (B) Fluorescence microscopy of DF-1 cells transfected with pEGFP-N3 vector (scale bar $=50 \mu \mathrm{m}$ or $25 \mu \mathrm{m}$ ). (C) $\mathrm{mRNA}$ fold-change of mRNA related with TLR3 response in DF-1 cells. mRNA fold-change was normalized to $\beta$-actin mRNA (D) Cell viability of DF-1 cells treated with Poly(l:C) for $24 \mathrm{~h}$. Data are expressed as mean $\pm S D(n=3)$. All data are expressed as mean $\pm S D(n=3)$. ${ }^{*} p<0.05,{ }^{* *} p<0.01,{ }^{* * *} p<0.001$, and ${ }^{\text {ns }} p>0.05$ by unpaired two-tailed Student's $t$-test. Poly (I:C), polyinosinic:polycytidylic acids; DAPI, 4',6-diamidino-2-phenylindole; TLR3, Toll-like receptors 3; TRAF3, TNF receptorassociated factor 3; TICAM1, Toll-like receptor adaptor molecule 1; NF-kB1, nuclear factor-kB1; TNFa, tumor necrosis factor $\alpha$; COX2, cyclooxygenase2; PPDPF, progenitor cell differentiation and proliferation factor.

DF-1 cells. The main functions of PPDPF in mammalians were well reported as a key regulator of development of pancreas in human. In contrast, the structure and function of PPDPF have not yet been reported in chickens.

In this study, we identified the potential for differences in TLR3 signaling and binding proteins in humans and chickens based on the amino acid sequence of TIR domain (Fig. 1). And we predicted the function and structure of PPDPF through bioinformatics (Fig. 2). The evolutionarily conserved domains present in chicken PPDPF proteins, the CLK2 kinase binding site, SH3 domain binding site, and ATP/GTP binding site, reveal the potential of PPDPF to participate in signaling pathways or act as enzymes. (Fig. 2A). The presence of $\mathrm{SH} 3$ domains in proteins suggests potential involvement in various signaling pathways by protein tyrosine kinases through protein- 
protein interactions [30]. CLK2 protein is implicated in a variety of signaling pathways, including regulation of inflammation and viral resistance [31,32] , and cell proliferation [33]. Presence of ATP/GTP binding site in protein, it can use the energy of ATP for enzymatic action [34]. The role of the chicken PPDPF domain should be verified through a loss of function study.

We examined the relationship between TLR3 signaling and PPDPF in chicken cells. The expression level of PPDPF is upregulated by TLR3 stimulation with Poly(I:C) in DF-1 cells but not in HD-11 cells (Figs. 3A and B). HD11 cells have low reactivity to exogenous dsRNA, and TLR3 activation is completed in a very short time, so PPDPF expression may not be induced even with poly(I:C) treatment [24,35]. TLR3 is mainly located on the cell surface of non-immune cells, including fibroblasts [36]. The DF-1 chicken embryo fibroblast cell line is derived from chicken embryos and has active TLR3 signaling pathways [37,38], demonstrating that DF-1 cells might be a suitable model to study the TLR3 responses in chickens.

It is of note that, in Poly(I:C) treated DF-1 fibroblasts, PPDPF-eGFP is mainly located in the nucleus (see Fig. 5B), and mechanism behind this localization remains to be studied, but at least, it seems that PPDPF may not have direct interaction with TLR3. Mechanism behind the localization of PPDPF remains to be studied. Overexpression of PPDPF reduced pro-inflammatory gene expression in DF-1 chicken embryonic fibroblasts. These results indicate the possibility of PPDPF acting as a negative regulator of TLR3 mediated inflammation in DF-1 chicken embryonic fibroblasts. NF- $\kappa \mathrm{B} 1$ can induce systemic pro-inflammatory gene expression under stress conditions, so inhibition of $N F-\kappa B 1$ expression by PPDPF could be effective in suppressing inflammation in poultry [39].

Future studies require evaluation of the effect of PPDPF protein on TLR3 signaling, inflammatory response, and binding protein through loss of function study of chicken PPDPF protein. Nevertheless, based on the sequence and predicted structure of the PPDPF protein and the study in PPDPF-overexpressing DF-1 cells, we suggest the possibility that PPDPF may participate in TLR3-mediated inflammatory responses in chickens.

\section{REFERENCES}

1. Bsibsi M, Ravid R, Gveric D, van Noort JM. Broad expression of Toll-like receptors in the human central nervous system. J Neuropathol Exp Neurol. 2002;61:1013-21. https://doi. org/10.1093/jnen/61.11.1013

2. Yao C, Oh JH, Lee DH, Bae JS, Jin CL, Park CH, et al. Toll-like receptor family members in skin fibroblasts are functional and have a higher expression compared to skin keratinocytes. Int J Mol Med. 2015;35:1443-50. https://doi.org/10.3892/ijmm.2015.2146

3. Akira S, Takeda K. Toll-like receptor signalling. Nat Rev Immunol. 2004;4:499-511. https:// doi.org/10.1038/nri1391

4. Karikó K, Buckstein M, Ni H, Weissman D. Suppression of RNA recognition by Tolllike receptors: the impact of nucleoside modification and the evolutionary origin of RNA. Immunity. 2005;23:165-75. https://doi.org/10.1016/j.immuni.2005.06.008

5. Chovatiya R, Medzhitov R. Stress, inflammation, and defense of homeostasis. Mol Cell. 2014;54:281-8. https://doi.org/10.1016/j.molcel.2014.03.030

6. Leung YHC, Nicholls JM, Ho CK, Sia SF, Mok CKP, Valkenburg SA, et al. Highly pathogenic avian influenza A H5N1 and pandemic H1N1 virus infections have different phenotypes in Tolllike receptor 3 knockout mice.J Gen Virol. 2014;95:1870-9. https://doi.org/10.1099/vir.0.066258-0

7. Stowell NC, Seideman J, Raymond HA, Smalley KA, Lamb RJ, Egenolf DD, et al. Long-term activation of TLR3 by poly (I:C) induces inflammation and impairs lung function in mice. 
Respir Res. 2009;10:43. https://doi.org/10.1186/1465-9921-10-43

8. Mora Izaguirre MO, Quester K, Petranovski V, González Dávalos L, Piña Garza E, Shimada Miyasaka A, et al. Effect of nanostructured zeolite with lipoic acid on performance and carcass yield in broiler. Rev Mex Cienc Pecu. 2018;9:185-202. https://doi.org/10.22319/rmcp.v9i2.4315

9. Guo ZY, Li JL, Zhang L, Jiang Y, Gao F, Zhou GH. Effects of alpha-lipoic acid supplementation in different stages on growth performance, antioxidant capacity and meat quality in broiler chickens. Br Poult Sci. 2014;55:635-43. https://doi.org/10.1080/00071668.2014.958057

10. Keestra AM, van Putten JPM. Unique properties of the chicken TLR4/MD-2 complex: selective lipopolysaccharide activation of the MyD88-dependent pathway. J Immunol. 2008;181:4354-62. https://doi.org/10.4049/jimmunol.181.6.4354

11. Jiang Z, Song J, Qi F, Xiao A, An X, Liu N, et al. Exdpf is a key regulator of exocrine pancreas development controlled by retinoic acid and ptf1a in zebrafish. PLOS Biol. 2008;6:e293. https://doi.org/10.1371/journal.pbio.0060293

12. Breunig M, Hohwieler M, Seufferlein T, Glöckner J, Liebau S, Kleger A. PPDPF impacts pancreatic progenitor cell formation derived from human pluripotent stem cell. Z Gastroenterol. 2018;56:e234. https://doi.org/10.1055/s-0038-1668739

13. Xiao Y, Lai Y, Yu Y, Jiang P, Li Y, Wang C, et al. The exocrine differentiation and proliferation factor (EXDPF) gene promotes ovarian cancer tumorigenesis by up-regulating DNA replication pathway. Front Oncol. 2021;11:669603. https://doi.org/10.3389/fonc.2021.669603

14. Vittrant B, Leclercq M, Martin-Magniette ML, Collins C, Bergeron A, Fradet Y, et al. Identification of a transcriptomic prognostic signature by machine learning using a combination of small cohorts of prostate cancer. Front Genet. 2020;11:550894. https://doi.org/10.3389/fgene.2020.550894

15. Ma N, Wang YK, Xu S, Ni QZ, Zheng QW, Zhu B, et al. PPDPF alleviates hepatic steatosis through inhibition of mTOR signaling. Nat Commun. 2021;12:3059. https://doi.org/10.1038/ s41467-021-23285-8

16. Blom N, Gammeltoft S, Brunak S. Sequence and structure-based prediction of eukaryotic protein phosphorylation sites.J Mol Biol. 1999;294:1351-62. https://doi.org/10.1006/jmbi.1999.3310

17. Walsh I, Di Domenico T, Tosatto SCE. RUBI: rapid proteomic-scale prediction of lysine ubiquitination and factors influencing predictor performance. Amino Acids. 2014;46:853-62. https://doi.org/10.1007/s00726-013-1645-3

18. McGuffin LJ, Adiyaman R, Maghrabi AHA, Shuid AN, Brackenridge DA, Nealon JO, et al. IntFOLD: an integrated web resource for high performance protein structure and function prediction. Nucleic Acids Res. 2019;47:W408-13. https://doi.org/10.1093/nar/gkz322

19. McGuffin LJ, Shuid AN, Kempster R, Maghrabi AHA, Nealon JO, Salehe BR, et al. Accurate template-based modeling in CASP12 using the IntFOLD4-TS, ModFOLD6, and ReFOLD methods. Proteins. 2018;86:335-44. https://doi.org/10.1002/prot.25360

20. Beug H, von Kirchbach A, Döderlein G, Conscience JF, Graf T. Chicken hematopoietic cells transformed by seven strains of defective avian leukemia viruses display three distinct phenotypes of differentiation. Cell. 1979;18:375-90. https://doi.org/10.1016/00928674(79)90057-6

21. Matsumoto M, Oshiumi H, Seya T. Antiviral responses induced by the TLR3 pathway. Rev Med Virol. 2011;21:67-77.https://doi.org/10.1002/rmv.680

22. Livak KJ, Schmittgen TD. Analysis of relative gene expression data using real-time quantitative PCR and the 2- $\Delta \Delta \mathrm{CT}$ method. Methods. 2001;25:402-8. https://doi.org/10.1006/ meth.2001.1262

23. Funami K, Matsumoto M, Oshiumi H, Akazawa T, Yamamoto A, Seya T. The cytoplasmic 'linker region' in Toll-like receptor 3 controls receptor localization and signaling. Int Immunol. 
2004;16:1143-54. https://doi.org/10.1093/intimm/dxh115

24. Karpala AJ, Lowenthal JW, Bean AG. Activation of the TLR3 pathway regulates IFN $\beta$ production in chickens. Dev Comp Immunol. 2008;32:435-44. https://doi.org/10.1016/ j.dci.2007.08.004

25. Cheng J, Sun Y, Zhang X, Zhang F, Zhang S, Yu S, et al. Toll-like receptor 3 inhibits Newcastle disease virus replication through activation of pro-inflammatory cytokines and the type-1 interferon pathway. Arch Virol. 2014;159:2937-48. https://doi.org/10.1007/s00705-014-2148-6

26. Wang Q, Miller DJ, Bowman ER, Nagarkar DR, Schneider D, Zhao Y, et al. MDA5 and TLR3 initiate pro-inflammatory signaling pathways leading to rhinovirus-induced airways inflammation and hyperresponsiveness. PLOS Pathog. 2011;7:e1002070. https://doi. org/10.1371/journal.ppat.1002070

27. Nailwal H, Chan FKM. Necroptosis in anti-viral inflammation. Cell Death Differ. 2019;26:413. https://doi.org/10.1038/s41418-018-0172-x

28. Jiang Z, Schatzmayr G, Mohnl M, Applegate TJ. Net effect of an acute phase responsepartial alleviation with probiotic supplementation. Poult Sci. 2010;89:28-33. https://doi. org/10.3382/ps.2009-00464

29. Anwar MA, Basith S, Choi S. Negative regulatory approaches to the attenuation of Toll-like receptor signaling. Exp Mol Med. 2013;45:e11. https://doi.org/10.1038/emm.2013.28

30. Kurochkina N, Guha U. SH3 domains: modules of protein-protein interactions. Biophys Rev. 2013;5:29-39. https://doi.org/10.1007/s12551-012-0081-z

31. Deshmukh V, O'Green AL, Bossard C, Seo T, Lamangan L, Ibanez M, et al. Modulation of the Wnt pathway through inhibition of CLK2 and DYRK1A by lorecivivint as a novel, potentially disease-modifying approach for knee osteoarthritis treatment. Osteoarthr Cartil. 2019;27:1347-60. https://doi.org/10.1016/j.joca.2019.05.006

32. Wong R, Balachandran A, Mao AYQ, Dobson W, Gray-Owen S, Cochrane A. Differential effect of CLK SR kinases on HIV-1 gene expression: potential novel targets for therapy. Retrovirology. 2011;8:47.https://doi.org/10.1186/1742-4690-8-47

33. Petsalaki E, Zachos G. Clks 1, 2 and 4 prevent chromatin breakage by regulating the Aurora B-dependent abscission checkpoint. Nat Commun. 2016;7:11451. https://doi.org/10.1038/ ncomms 11451

34. Chauhan JS, Mishra NK, Raghava GPS. Identification of ATP binding residues of a protein from its primary sequence. BMC Bioinformatics. 2009;10:434. https://doi.org/10.1186/14712105-10-434

35. Peroval MY, Boyd AC, Young JR, Smith AL. A critical role for MAPK signalling pathways in the transcriptional regulation of toll like receptors. PLOS ONE. 2013;8:e51243. https://doi. org/10.1371/journal.pone.0051243

36. Matsumoto M, Funami K, Tanabe M, Oshiumi H, Shingai M, Seto Y, et al. Subcellular localization of Toll-like receptor 3 in human dendritic cells. J Immunol. 2003;171:3154-62. https://doi.org/10.4049/jimmunol.171.6.3154

37. Tian W, Zhao C, Hu Q, Sun J, Peng X. Roles of Toll-like receptors 2 and 6 in the inflammatory response to Mycoplasma gallisepticum infection in DF-1 cells and in chicken embryos. Dev Comp Immunol. 2016;59:39-47. https://doi.org/10.1016/j.dci.2016.01.008

38. Foster D. Development of a spontaneously immortalized chicken embryo fibroblastic cell line. Virology. 1998;248:305-11.

39. Surai PF, Kochish II, Kidd MT. Redox homeostasis in poultry: regulatory roles of NF- $\mathrm{kB}$. Antioxidants. 2021;10:186. https://doi.org/10.3390/antiox10020186 